\title{
Aberration-corrected STEM of Highly Loaded Pt 1 /NiO Single-atom Catalysts: Structural and Catalytic Stability
}

\author{
S. B. Duan ${ }^{1,2}$ R. M. Wang ${ }^{2}$ and J. Y. Liu ${ }^{1}$ \\ 1. Department of Physics, Arizona State University, Tempe, Arizona 85287, USA \\ 2. Department of Physics, Beihang University, Beijing 100191, China
}

Supported precious metal catalysts play a significant role in many chemical transformations including energy generation and control of toxic emissions. Precious metals are, however, expensive and thus efficient usage of precious metals is of critical importance for practical applications. The development of single-atom catalysts (SACs), which maximize the efficiency of costly active components of supported metal catalysts, is important for both fundamental studies and industrial applications [1-3]. The issue with the current SACs is that they usually consist of extremely low loading levels of metal, resulting in low specific activity and conversion rate. High loading levels of isolated single metal atoms are not stable and sinter to larger particles during catalytic reactions. Stabilization of single metal atoms by anchoring sites, for example, surface/subsurface defects, hydroxyl groups, and structural stabilizers, becomes imperative for further development of practical SACs.

The high loading Pt SACs were synthesized by a modified adsorption method. Briefly, $\mathrm{H}_{2} \mathrm{PtCl}_{6}$ solution was used as the Pt precursor and was mixed with the pre-formed $\mathrm{NiO}$ nanocrystallites. The nominal loading of the Pt metal was $2.0 \mathrm{wt} \%$. The resultant precipitate was filtered, washed, dried and calcined at $400^{\circ} \mathrm{C}$ for 5 hours (denoted as $2 \mathrm{Pt}_{1} / \mathrm{NiO}$ ). The catalytic performance of the synthesized catalysts for $\mathrm{CO}$ oxidation was evaluated in a fixed-bed reactor. The feed gas composition was $1 \mathrm{vol} \% \mathrm{CO}+1 \mathrm{vol}_{2} \mathrm{O}_{2}$ and balance He with a flow rate of $33 \mathrm{ml} / \mathrm{min}$, and $80 \mathrm{mg}$ catalyst was directly used without reduction. The outlet gas compositions were on-line analyzed by a gas chromatograph and the CO conversion was calculated based on the inlet and outlet CO concentrations. Aberration-corrected high-angle annular dark-field (AC-HAADF) microscopy was used to characterize the synthesized and used catalysts.

The low magnification image of Figure 1a clearly shows that there were no Pt particles in the assynthesized $2 \mathrm{Pt}_{1} / \mathrm{NiO}$ catalyst. The atomic resolution image of Figure $1 \mathrm{~b}$ reveals only isolated $\mathrm{Pt}$ single atoms uniformly dispersed onto the surfaces of $\mathrm{NiO}$ nanocrystallites. By analyzing many such low and high magnification images of various regions of the as-synthesized catalyst, we unambiguously concluded that the as-synthesized $2 \mathrm{Pt}_{1} / \mathrm{NiO}$ catalyst contained only isolated single Pt atoms. Figure 2 displays $\mathrm{CO}$ conversion versus temperature and time-on-stream for the $2 \mathrm{Pt}_{1} / \mathrm{NiO}$ catalyst. Except the initial increase of the $\mathrm{CO}$ conversion (Figure $2 \mathrm{~b}$ ), the $2 \mathrm{Pt}_{1} / \mathrm{NiO}$ did not deactivate at all during the 1,000 minutes test for $\mathrm{CO}$ oxidation reaction at 500K. Figure 1c and 1d display the AC-HAADF images of the used catalyst, which contained only individually isolated Pt single atoms. Thus we have developed a stable, high number density $\mathrm{Pt}_{1} / \mathrm{NiO} \mathrm{SAC}$ for $\mathrm{CO}$ oxidation. Similar synthesis strategies will be used for developing other types of SACs for various types of catalytic reactions.

\section{References:}

[1] Qiao, B. et al, Nature Chem. 3, (2011), p. 634.

[2] Moses-DeBusk, M., et al, J Am. Chem. Soc. 135, (2013), p. 12634.

[3] Yang, M., et al, Science 346, (2014), p. 1498.

[4] The authors acknowledge the College of Liberal Arts and Sciences of Arizona State University for funding and the use of the John M. Cowley CHREM facilities at Arizona State University. 

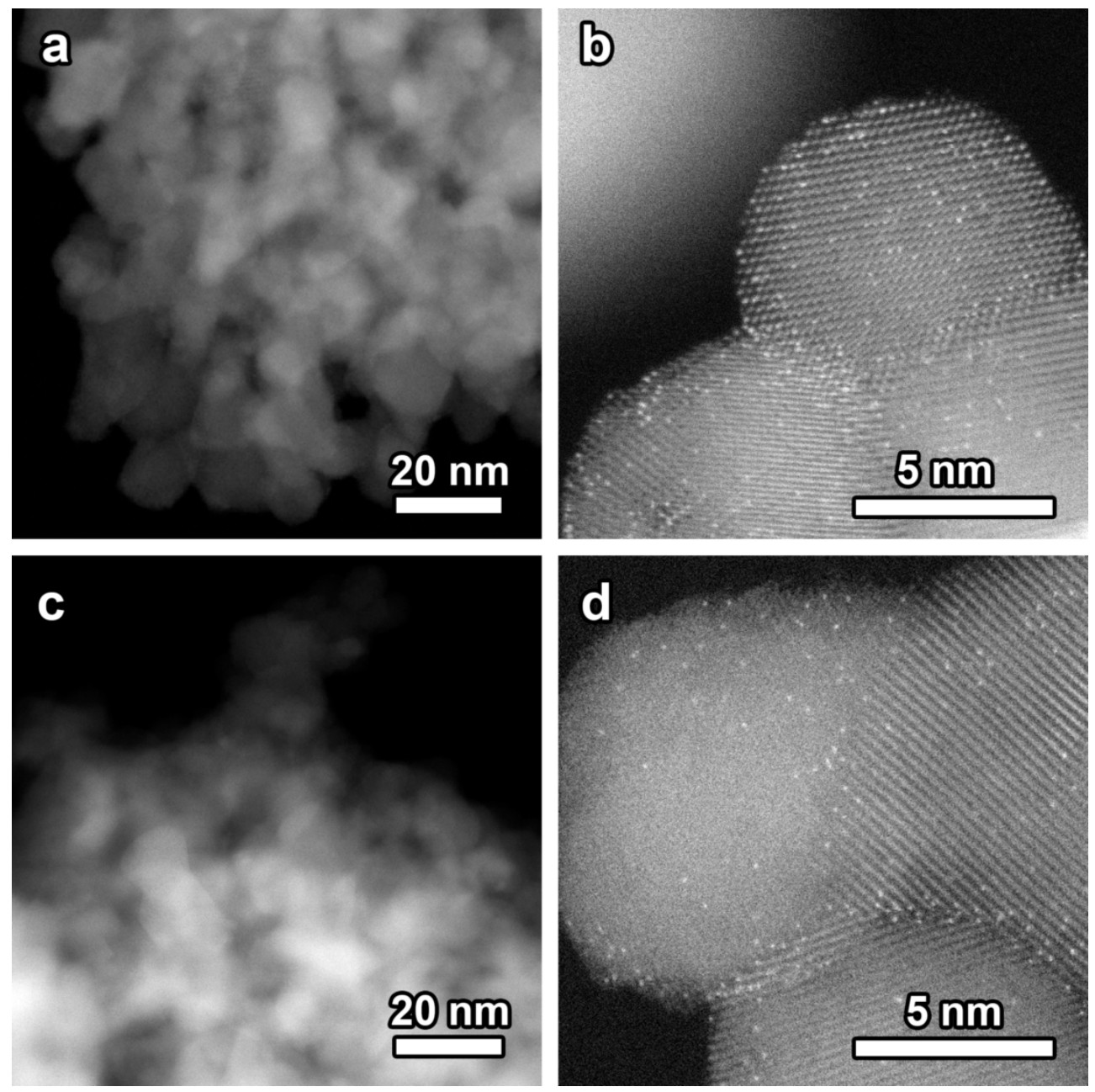

Figure 1. Low and high magnification AC-HAADF images of (a, b) fresh $2 \mathrm{Pt}_{1} / \mathrm{NiO} \mathrm{SAC}$ and (c, d) after $\mathrm{CO}$ oxidation for 1,000 minutes at $500 \mathrm{~K}$, revealing that the $2 \mathrm{Pt}_{1} / \mathrm{NiO}$ catalyst consisted of only isolated single Pt atoms which were stable during the $\mathrm{CO}$ oxidation reaction.
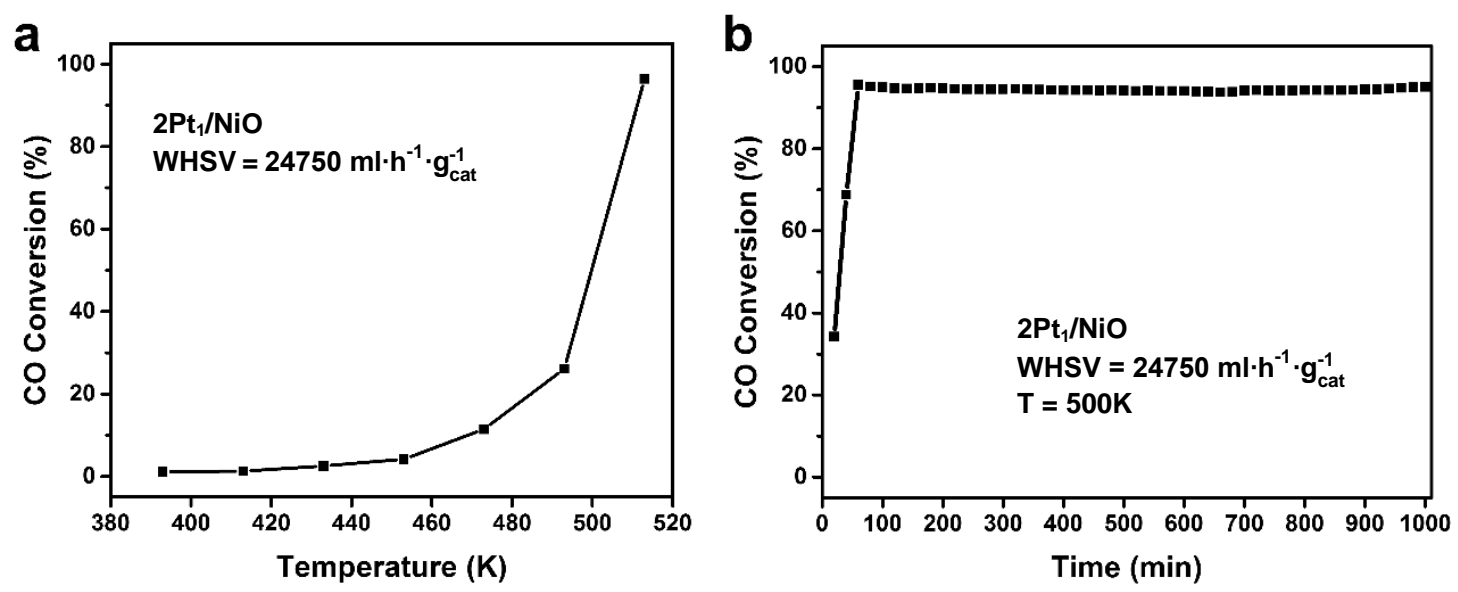

Figure 2. $\mathrm{CO}$ conversion vs (a) temperature and (b) time-on-stream $(\mathrm{T}=500 \mathrm{~K})$ for the $2 \mathrm{Pt}_{1} / \mathrm{NiO}^{\mathrm{SAC}}$, clearly demonstrating the stability of the as-synthesized $2 \mathrm{Pt}_{1} / \mathrm{NiO} \mathrm{SAC}$. 